\title{
Strategic Thinking and Development Opportunity for a Comprehensive Healthcare Industry based on Traditional Chinese Medicine
}

\author{
Zhang Boli ${ }^{1,2}$, Zhang Junhua ${ }^{1}$, Chen Shilin ${ }^{2}$, Duan Jin'ao ${ }^{3}$, Huang Luqi ${ }^{2}$, Sun Xiaobo ${ }^{4}$, Tu Pengfei ${ }^{5}$, Ye Zuguang ${ }^{2}$,

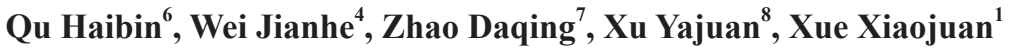 \\ 1. Tianjin University of Traditional Chinese Medicine, Tianjin 300193, China \\ 2. China Academy of Chinese Medical Sciences, Beijing 100700, China \\ 3. Nanjing University of Chinese Medicine, Nanjing 210023, China \\ 4. Chinese Academy of Medical Sciences, Beijing 100193, China \\ 5. Peking University, Beijing 100871, China \\ 6. Zhejiang University, Hangzhou 310058, China \\ 7. Changchun University of Chinese Medicine, Changchun 130117, China \\ 8. Jilin Academy of Chinese Medicine, Changchun 130012, China
}

\begin{abstract}
With China's recent economic and social developments, the demand for healthcare services has been increasing rapidly. Traditional Chinese Medicine (TCM) is a unique healthcare resource, in addition to being an economic resource, with great potential in China. The services provided by TCM include health preservation, healthcare, medical treatment, rehabilitation, and so forth, while the core of traditional medicine supplies healthcare services based on traditional Chinese medicinal products. The modernization of TCM will promote the formation of a comprehensive healthcare industry based on TCM that involves the cultivation, R\&D, production, circulation, and sales of traditional Chinese medical products, which are part of a long chain of industry that crosses both sectors and regions. This has strong advantages that include the adjustment of industrial structures, the ability to increase farmers' incomes and employment, services that will work toward healthcare reform, benefits for people's livelihoods, and ecological protection. This paper expounds on the development situation of a comprehensive healthcare industry based on TCM, analyzes existing problems and shortcomings, and proposes one strategic center (meeting the needs of healthcare services), two strategic priorities (planning and supervision; the advancement of quality and effectiveness), four development directions (good agricultural practice; new product $\mathrm{R} \& \mathrm{D}$; commercial and trade logistics; international development), and six key tasks (policy; supervision; logistics system, quality of production, base and brand; overseas business).
\end{abstract}

Keywords: comprehensive healthcare; comprehensive healthcare industry; traditional Chinese medicine; modernization of traditional Chinese medicine

\section{Introduction}

Health is the foundation and the essential condition for the comprehensive development of humanity. The health industry is, on one hand, related to people's well-being and livelihood, and on the other hand, related to economic development. With social and economic development, people's demands for health services are rising increasingly. There is an enormous market

Received date: February 10, 2017; Revised date: February 20, 2017

Corresponding author: Zhang Boli, Tianjin University of Traditional Chinese Medicine, China Academy of Chinese Medical Sciences, Professor; Chinese Academy of Engineering, Academician. Major research fields include Chinese medicine for cardiovascular disease and modernization of TCM. E-mail: zhangbolipr@163.com Funding program: CAE Advisory Project “Development Strategy for Comprehensive Healthcare Industry of Traditional Chinese Medicine” (2013-XZ-19)

Chinese version: Zhang Boli et al. Strategic Thinking and Development Opportunity for a Comprehensive Healthcare Industry based on Traditional Chinese Medicine. Strategic Study of CAE, https://doi.org/10.15302/J-SSCAE-2017.02.003 
demand, as well as fantastic development opportunities, for the healthcare industry, in particular regarding the change of the disease spectrum, the arrival of an aging society, the change of people's lifestyles, and the unceasing pursuit of better health. The healthcare industry is an important part of the modern service industry. Currently, the industry outputs account for only about $5 \%$ of Gross Domestic Product in China, but in developed countries, it accounts for more than $10 \%$ of their GDP. China's healthcare industry has great potential for development [1].

Traditional Chinese Medicine (TCM) is a unique health resource of China; it also represents an economic resource with enormous potential. TCM health services mainly include health maintenance, healthcare, medical treatment, and rehabilitation, but its core is the supply of TCM-related products. With the advancement and modernization of TCM, the scientific research platform and research level of TCM have been raised, promoting its progress, in particular with the gradual formation of a comprehensive healthcare industry based on TCM with Chinese medicine manufacturing as its foundation.

This type of comprehensive healthcare industry is a new industry, with TCM industry as its main part, TCM agriculture as its base, TCM business as its hub, and TCM knowledge innovation as its momentum. It has formed a cross-industry, cross-region, and cross-border TCM industry chain that includes TCM-related product R\&D, manufacturing, circulation, and sales $[2,3]$. The comprehensive healthcare products of TCM include Chinese patent medicine, healthcare products, Chinese medicinal materials, Chinese medicine decoction pieces, as well as extractive, healthy food and drinks, cosmetics, household chemicals, veterinary drugs, animal feed prepared with Chinese herbs, and manufacturing equipment [2-4]. Developing a comprehensive healthcare industry based on TCM can bring about wide-ranging advantages such as readjusting the industrial structure, increasing employment, increasing farmers' incomes, enhancing medical reform, benefiting people's livelihoods, and environmental protection, among others.

Under the support of the consulting project of the Chinese Academy of Engineering, and through extensive investigation and analysis, we have summarized the current development status of a comprehensive healthcare industry based on TCM. We have thus discovered four major problems hindering this development; put forward strategic thinking, strategic objectives, and strategic foci for its development; and proposed six countermeasures and suggestions with a view toward better promotion and realization of the sustainable development of this type of comprehensive healthcare industry.

\section{Development opportunity for a comprehensive healthcare industry based on TCM}

China is a vast country with more than 1.3 billion people. With the development of our country's economy and society, we have seen the construction of a healthy China and progress toward building a moderately prosperous society in all respects. The masses' desire for health, and their desire to maintain that health, are increasingly becoming intensified; this has created development conditions and a massive market for the rapid development of the healthcare industry. Professor Tu Youyou won the 2015 Nobel Prize in physiology or medicine for discovering Artemisinin (a malaria treatment drug); this has enabled TCM to draw increasing attention and importance from home and abroad, and the world's demand for TCM has been continuously growing.

In recent years, the development of TCM has obtained strong support from the Party Central Committee and the State Council. Consequently, the Traditional Chinese Medicine Health Service Development Planning (2015-2020), the Traditional Chinese Medicinal Materials Protection and Development Planning (2015-2020), and the Outline of the Strategic Plan on the Development of Traditional Chinese Medicine (2016-2030), along with other documents, have been issued. Furthermore, the promulgation of the Law of the People's Republic of China on Traditional Chinese Medicine provides legal protection and policy support for the development of a comprehensive healthcare industry based on TCM. In the process of promoting the revitalization of TCM, the comprehensive healthcare industry based on TCM will continue to grow, and it will become an important force in the construction of a healthy China.

\section{Current development status of the comprehensive healthcare industry based on TCM}

Currently, 2088 pharmaceutical enterprises have been accredited by the good manufacture practice to manufacture Chinese patent medicines; of those, more than 30 enterprises' market value exceeds 10 billion yuan [5]. There are nearly 500 key Chinese patent medicines whose annual sale exceeds 100 million yuan [6]; the industrial output of Chinese medicines has been steadily increasing, to 786.7 billion yuan in 2015 from 23.54 billion yuan in 1996 , which accounted for $28.55 \%$ of the pharmaceutical industry's total output [7].

There are a total of 12807 varieties of medicinal materials, and more than 1200 varieties of commercial medicinal materials, of which 600 varieties belong to common medicinal materials [3], and more than 300 varieties are for artificial herbal medicine plants. Nationwide, the area devoted to medicinal herb planting exceeds 50 million $\mathrm{mu}\left(1 \mathrm{mu} \approx 666.67 \mathrm{~m}^{2}\right)$. The rich diversity in medicinal materials and extensive planting area provide a resource guarantee for the development of a comprehensive healthcare industry based on TCM [5]. In 2014, enterprises developed around TCM decoction pieces acquired a sales income of 150 billion yuan. The market for TCM-derived daily chemical products and TCM-derived cosmetics has grown rapidly; in 2010, the market demand for daily chemical products containing 
TCM or plants amounted to 61 billion yuan, and in 2014, that same market demand reached 110 billion yuan. Sales in the healthcare market increased from 280 billion yuan in 2012 to 400 billion yuan in 2014, of which 300 billion yuan was from healthcare products and healthcare food containing TCM; the average annual growth rate reached $10 \%-15 \%$. TCM-based veterinary medicines has also rapidly expanded; its sales in 2014 reached 20 billion yuan. In 2014, plant-derived pesticides sales came to about 2 billion yuan, and TCM processing instruments and equipment sales scale exceeded 40 billion yuan. In 2014, TCM circulation industry sales reached 300 billion yuan [8]. Each year, the TCM industry in China uses about $7 \times 10^{5}$ t of medicinal plant materials; annually, there are millions of tons of non-medicinal parts of plants generated. Consequently, the TCM circulation economy has a brilliant future and can accomplish great deeds. Its annual output value is estimated to reach 50 billion yuan $[9,10]$.

On the basis of the above analysis, the comprehensive healthcare industry based on TCM, including TCM industry, TCM agriculture, TCM commerce, TCM food, TCM healthcare products, TCM daily chemical products, TCM equipment, and other TCM-related commercial activities and products, has developed rapidly and secured outstanding achievements. Its output value increased from over 700 billion yuan in 2009 to 1.2 trillion yuan in 2013, demonstrating rapid growth momentum, and it is expected to reach 3 trillion yuan in 2020, becoming an important pillar of the healthcare industry in China [11].

The Belt and Road Initiative has given a strong impetus to the development of the TCM health industry and its international trade growth. According to the statistics of China Customs, the import and export volume of China's TCM related products totaled 4.63 billion dollars in 2014, rising $9.79 \%$ year on year; of that, the export volume totaled 3.592 billion dollars, rising $14.49 \%$ year on year. In 2014, the trade volume between China and those countries and regions along the Belt and Road totaled 2.543 billion dollars. That same trade volume was 781 million dollars in 2008; it rose by $226 \%$ [12]. In March 2012, departments including the Commerce Department and State Administration of Traditional Chinese Medicine, as well as several others, jointly unveiled "Several Advices on Promoting the Development of Traditional Chinese Medicine Service Trade," which will play an important role in promoting the continuous expansion of the international market of TCM healthcare products.

\section{Problems facing the development of a comprehensive healthcare industry based on TCM}

\subsection{Lack of top-level design and regional planning}

Currently, the comprehensive healthcare industry based on TCM is still in its early stages of development, and its devel- opment mainly originates from the spontaneous behavior of various enterprises. Although the country has enacted measures including TCM Healthcare Industry Development Planning and TCM Material Protection and Development Planning, the lack of unified planning for the comprehensive healthcare industry chain based on TCM has led to blind development across different regions. As a result of the failure to combine different features of regional development, regional advantages of the comprehensive healthcare industry cannot be formed, leading to serious low-level redundant development.

\subsection{Lack of rational support policies and administrative measures}

The policies and support systems related to the comprehensive healthcare industry based on TCM are far from perfect; some policies and administrative measures have even constrained its development. For example, quality improvement based on the implementation of secondary development of TCM requires the optimization of previous unreasonable processes and standards as well as of the range of clinical applications, but it lacks the support of corresponding policies. Furthermore, the lack or lagging of administrative measures for the development of a TCM circulation economy, healthcare products/food, and cosmeceuticals has hindered the industry's development.

\subsection{Weak links in the chain of the comprehensive healthcare industry based on TCM}

The comprehensive healthcare industry based on TCM involves TCM agriculture, industry, commerce, logistics and other fields, and its main components are TCM materials, Chinese patent medicines, healthcare products and food. However, TCM derived cosmetics, TCM derived pesticides, TCM derived veterinary medicine, TCM feed, TCM manufacturing equipment, TCM international trade, and the TCM circulation economy are still in the early stages of development. Although they have enormous development potential and space, they lack planning and support, becoming the weak links in the market expansion of the comprehensive healthcare industry based on TCM.

Commercially, the circulation channel of the comprehensive healthcare industry based on TCM is still mainly based on traditional modes with miscellaneous intermediate links, and the incomplete product quality tracing system increases the circulation cost. The circulation system of TCM health products is based on the internet; the internet of things and big data need to be strengthened.

\subsection{Weak scientific research of TCM healthcare products leads to inferior development}

At present, TCM healthcare product research and develop- 
ment is challenged by low levels of investment, a lack of independent research and development of technology platforms and professionals, and low-level product redundant development, for example. Apart from medicine, other TCM healthcare products mainly add low value and are quickly introduced into the market but are short-lived due to the lack of strict quality control. The lack of in-depth and intensive research in product $\mathrm{R} \& \mathrm{D}$, quality standards, processes, and packaging lead to low quality and low-value added products, failing to satisfy consumers' demands for high quality products. In addition, the development of healthcare products is blind, deviates from public health requirement, and lacks effective and safe research data, resulting in low consumer recognition.

\subsection{TCM healthcare products have weak international competitiveness}

Due to cultural differences and the barrier of policies and regulations, TCM products are not yet in line with the standards and requirements of the international pharmaceutical market. They still face competition from Japan, Germany, America, France, and other countries. According to China Customs statistics, of all the exported TCM products in 2014, the export value of plant extract amounted to 1.777 billion dollars, TCM materials and TCM decoction pieces amounted to 1.295 billion dollars, healthcare products amounted to 270 million dollars, and Chinese patent medicines amounted to 250 million dollars. It can thus be seen that the export products were mainly low-value added raw materials and extracts.

\section{Development strategy for a comprehensive healthcare industry based on TCM}

\subsection{Strategic objectives}

By 2030, a comprehensive healthcare industry based on TCM with a reasonable structure, reliable quality, and prominent efficiency and that matches the revitalization and development of TCM, as well as covering the entire life cycle, shall be established. The industry scale will reach 5 trillion yuan and meet the basic health service requirements of the broad population. A relatively perfect policy system and management service system shall be formed to realize comprehensive regional coordinated development and the delivery of success, as well as the advancement of quality and effectiveness and the diversified, high-end and international development of the comprehensive healthcare industry. A batch of well-known brand products and brand enterprises of the comprehensive healthcare industry with relatively strong international competitiveness shall be established, and, furthermore, the international market share, international status, and influence of TCM products shall be further increased. A health industry based on TCM shall become an important part of the country's emerging industries, and TCM's contribution proportion to China's economic and social development shall be further increased.

\subsection{Strategic foci}

In the coming period, the development strategy of a comprehensive healthcare industry based on TCM can be generalized as follows: one strategic center (meeting the needs of healthcare service); two strategic priorities (planning and supervision; the advancement of quality and effectiveness); four development directions (good agriculture practice; new product R\&D; commercial and trade logistics; international development).

\subsection{Key tasks}

(1) Improve the policy system for the development of a comprehensive healthcare industry based on TCM. Adhere to the governmental guidance and support and give full play to market vitality. Enhance the implementation of the function of government such as through policy formulation, system guarantees, implementation supervision, and planning development, in line with the development requirements of a comprehensive healthcare industry based on TCM, and study and solve system and mechanism barriers that exist in the process of development.

(2) Build a supervision system for the comprehensive healthcare industry based on TCM. Take enthusiastic advantage of modern scientific and technological methods to strengthen the industry's self-regulation and social supervision. Bring industry associations in, encouraging them to play important roles in organization and coordination, industry counseling, standard setting, surveillance research, talent cultivation, and third-party evaluation, among other activities, to safeguard industry reputation and guarantee development.

(3) Build a modern logistics system for the comprehensive healthcare industry based on TCM. Set up a modern, normalized, standard, and intensive TCM commercial circulation system, and build a batch of exemplary TCM commercial and logistics centers that take on leading roles. Take advantage of network technology, develop internet and TCM e-commerce platforms, and establish and improve TCM sales networks to provide commercial communication platforms and information network services for the planting, processing, production, and sales of TCM materials.

(4) Start a project for "advancing quality and benefit." The prominent problems that hinder the development of a comprehensive healthcare industry based on TCM are "quality" and "benefits." TCM healthcare products need to meet high standards and strict quality criteria. The market competitiveness enhancement and quality improvement of TCM comprehensive healthcare products mainly depends on science and technology. Therefore, the project of "Advancing Quality and Effectiveness" 
shall be implemented through the use of intensified scientific and technological support with a view to systematically improving comprehensive healthcare product quality and industrial benefits, as well as by realizing the coordination and unification of their development and protection.

(5) Start demonstration bases and brand projects. Strengthen their overall arrangement and guidance, and build national and regional demonstration bases for the comprehensive healthcare industry based on TCM. This is beneficial to integrate advantageous resources and make the industry bigger and stronger, and at the same time, this process can play a demonstrative role, producing a radiating and driving effect, promoting mutual improvement, and cultivating well-known brands and enterprises.

(6) Start overseas expansion projects. Adapt development thinking, and expand markets in the countries along the Belt and Road. Support a batch of TCM healthcare products with prominent market strength and high quality in the overseas market, create several TCM service trade demonstration regions with powerful comprehensive strength and prominent international influence, and enlarge the international market share and enhance the awareness of TCM health products.

In his book, The New Wellness Revolution: How to Make a Fortune in the Next Trillion Dollar Industry, American economist Paul Plizer named the health industry, following the IT industry, the world's "fifth wave of wealth." This shows that the health industry will become the industrial pillar for promoting the development of the global economy. A comprehensive healthcare industry based on TCM is a manifestation of "prevention, cure, and maintenance," and it has become a new commerce type that is oriented by people's improved livelihoods. The development of a comprehensive healthcare industry based on TCM, the strengthening of the TCM industry, the creation of bigger TCM-related industries, and the construction of modern TCM intellectual property platforms and commercial logistics systems has certain benefits. It will upgrade the development scale and level of the TCM industry, promote the protection and utilization of TCM resources, and play an important role in fostering the innovation ability of national medical industry. This will promote the development of a health industry with distinct Chinese characteristics, serving the construction of a healthy China.

\section{References}

[1] NDRC. Interpretation of several opinions on promoting the development of health-service industry [J]. Science \& Technology Industry of China, 2013(10): 14-17. Chinese.

[2] Zhang B L. Support and promote the development of healthy industry of traditional Chinese medicine [J]. China Food Drug Administration, 2010(5): 8-9. Chinese.

[3] Huang L Q, Li J D, Li Z, et al. The situation and problems of the development of modern Chinese medicine industry chain in China [J]. China Venture Capital, 2010, 3(5): 67-69. Chinese.

[4] Li Y W, Cui M, Zhao Y K, et al. Strengthening information Industrialization of traditional Chinese medicine and promoting the healthy industry development of greater traditional Chinese medicine [J]. China Pharmacy, 2010: 577-579. Chinese.

[5] http://www.scio.gov.cn/zxbd/wz/ Document/1534697/1534697. htm. China State Council Information Office of the PRC. White Paper: Traditional Chinese Medicine in China [R]. (2016-12-06) [2016-12-15]. Chinese.

[6] South Institute of Economic Information. Industry report of traditional Chinese medicine in China [R]. Guangzhou: South Institute of Economic Information, 2015. Chinese.

[7] Zhang B L, Chen C H. Modernization of Chinese medicine for twenty years [M]. Shanghai: Shanghai Science and Technology Press, 2016. Chinese.

[8] Zhang B L. Research report on development strategy for great health industry of traditional Chinese medicine [R]. Tianjin: Tianjin University of Traditional Chinese Medicine, 2015. Chinese.

[9] Zhu H X, Duan J A, Guo L W, et al. Optimization theory and practical application of membrane science technology based on resource of traditional Chinese medicine residue [J]. China Journal of Chinese Materia Medica, 2014, 39(9): 1728-1732. Chinese.

[10] Duan J A, Su S L, Guo S, et al. Research on resources chemistry of Chinese medicinal materials and resources recycling utilization ways and goals and tasks [J]. China Journal of Chinese Materia Medica, 2015, 40(7): 3395-3401. Chinese.

[11] Zhang B L, Zhang J H. Twenty years' review and prospect of modernization research on traditional Chinese medicine [J]. China Journal of Chinese Materia Medica, 2015, 40(17): 3331-3334. Chinese.

[12] Xu H Q. Thinking of the developing strategy of Chinese medicine trade under the background of "The Belt and Road" [J]. Northern Economy, 2015(5): 38-40. Chinese. 